ARTICLE

Received 18 Aug 2014 | Accepted 2 Dec 2014 | Published 27 Jan 2015 | Updated 25 Feb 2015

DOI: $10.1038 /$ ncomms 7021

OPEN

\title{
Single-photon sensitive light-in-flight imaging
}

\author{
Genevieve Gariepy ${ }^{1}$, Nikola Krstajić ${ }^{2,3}$, Robert Henderson ${ }^{2}$, Chunyong Li', Robert R. Thomson', \\ Gerald S. Buller ${ }^{1}$, Barmak Heshmat ${ }^{4}$, Ramesh Raskar ${ }^{4}$, Jonathan Leach ${ }^{1}$ \& Daniele Faccio ${ }^{1}$
}

The ability to record images with extreme temporal resolution enables a diverse range of applications, such as fluorescence lifetime imaging, time-of-flight depth imaging and characterization of ultrafast processes. Recently, ultrafast imaging schemes have emerged, which require either long acquisition times or raster scanning and have a requirement for sufficient signal that can only be achieved when light is reflected off an object or diffused by a strongly scattering medium. Here we present a demonstration of the potential of single-photon detector arrays for visualization and rapid characterization of events evolving on picosecond time scales. The single-photon sensitivity, temporal resolution and full-field imaging capability enables the observation of light-in-flight in air, as well as the measurement of laser-induced plasma formation and dynamics in its natural environment. The extreme sensitivity and short acquisition times pave the way for real-time imaging of ultrafast processes or visualization and tracking of objects hidden from view.

\footnotetext{
${ }^{1}$ Institute of Photonics and Quantum Sciences, Heriot-Watt University, David Brewster Building, Edinburgh EH14 4AS, UK. ${ }^{2}$ Institute for Micro and Nano Systems, University of Edinburgh, Alexander Crum Brown Road, Edinburgh EH9 3FF, UK. ${ }^{3}$ Queen's Medical Research Institute, University of Edinburgh, 47 Little France Crescent, Edinburgh EH16 4TJ, UK. ${ }^{4}$ Camera Culture, MIT Media Lab, MIT, 75 Amherst Street, E14-474, Cambridge, Massachusetts 02139, USA. Correspondence and requests for materials should be addressed to G.G. (email: gg132@hw.ac.uk) or to D.F. (email:d.faccio@hw.ac.uk).
} 
ight was first captured in its flight by Abramson in 1978 (ref. 1), who used a holographic technique to record the wavefront of a pulse propagating and being scattered by a white-painted screen placed in its path. This high-speed recording technique allowed the dynamic observation of light phenomena like reflection, interference and focusing that are normally observed statically ${ }^{2,3}$. More recently, light-in-flight holography has been performed in a scattering medium rather than using a reflective screen ${ }^{4,5}$. Light can also be captured in motion in a scattering medium using a streak camera that has picosecond temporal resolution, thus removing the need for interferometry and coherent illumination but requires additional hardware to raster scan the two-dimensional (2D) scene, which increases the acquisition time to hours ${ }^{6,7}$. A few other techniques possess the temporal resolution to observe light in motion as it illuminates a scene, such as photonic mixer devices based on modulated illumination, albeit with a temporal resolution limited to a few nanoseconds ${ }^{8}$. Alternatively, time-encoded amplified imaging can record images at the repetition rate of a laser by exploiting wavelength-encoded illumination of a scene and amplified detection through a dispersive fibre, albeit with $160 \mathrm{~ns}$ temporal and spatial resolution' ${ }^{9}$. Recent studies based on computer tomography using data from multiple probe pulses enabled reconstruction of picosecond pulse propagation phenomena in condensed media ${ }^{10}$. The current challenge is to simplify data acquisition and reduce acquisition times by achieving full imaging capability and low-light sensitivity while maintaining temporal resolution in the picosecond regime.
Here we address this challenge and provide an imaging solution that simultaneously acquires spatial and temporal information $(x, y$ and $t)$. The technology we use is based upon a two-dimensional Silicon CMOS array of single-photon avalanche diode (SPAD) detectors with each individual pixel operated in time-correlated single-photon counting (TCSPC) mode ${ }^{11-14}$. Individual SPAD detectors are increasingly used throughout the field of optics; in particular, their high temporal resolution has made them useful for single-photon time-of-flight measurements $^{15,16}$, fluorescence lifetime imaging ${ }^{14}$ and photon counting ${ }^{17}$. Recent advances in electronics have enabled the development of arrays of SPADs, giving the capability to perform light-in-flight measurements. In these experiments, we use a $32 \times 32$-pixel array that has single-photon sensitivity and acquires time information with a resolution of $67 \mathrm{ps,} \mathrm{which} \mathrm{provides} \mathrm{the}$ ability to freeze the motion of light with a blurring of only a few centimeters $^{18}$.

\section{Results}

Experimental setup. The setup for our experiment is illustrated in Fig. 1. The scene is imaged onto the SPAD array using a fisheye lens. Laser pulses propagate at $4 \mathrm{kHz}$ from right to left across the field of view and are reflected by two mirrors. Light from the laser pulses is scattered by air molecules and then detected by the SPAD camera.

The SPAD camera is operated in TCSPC mode: every pixel has its own picosecond timer. The timer is started by the detection of
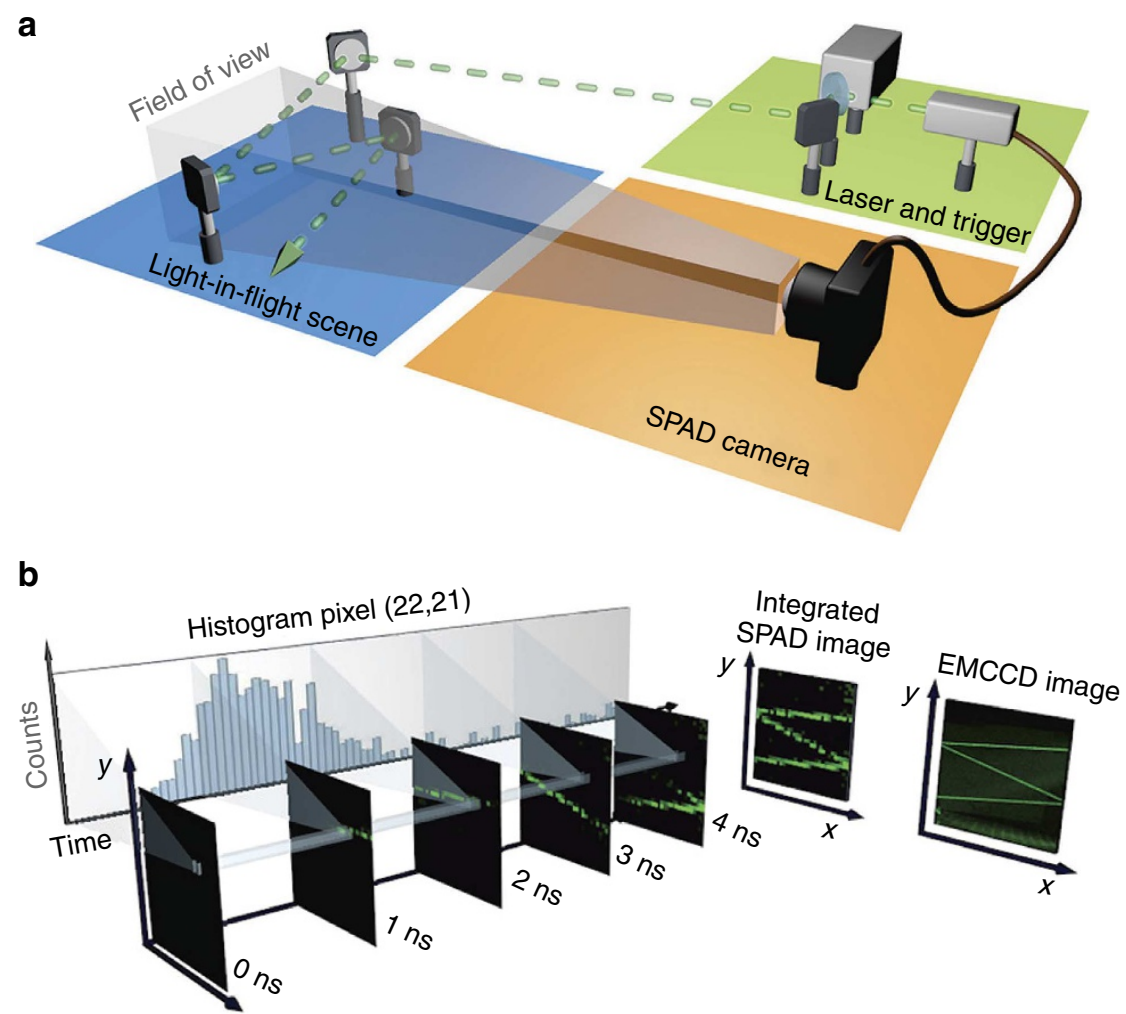

Figure 1 | Light-in-flight measurement and data cube. (a) A laser pulse is reflecting off multiple mirrors, passing three times across the field of view of the SPAD camera $\left(35 \times 35 \mathrm{~cm}^{2}\right)$. The same laser is used to create a trigger sent to the camera. The SPAD camera collects scattered photons from the laser pulse. The field of view does not contain the mirrors because the scattered light coming from the mirror surfaces is much more intense than the Rayleigh-scattered light during propagation. (b) The histogram indicates the time of arrival of the laser pulse as measured by pixel (22, 21). The time frames, shown at 0, 1, 2, 3 and 4 ns, show the evolution of the pulse in time as it propagates across the scene. The integration of all frames gives the total path followed by the light, similarly to what can be acquired by an EMCCD camera at maximum gain for an exposure time of 7 s. 
a single photon, and stopped by the arrival of a periodic trigger, which is derived from the laser pulses. The time between start and stop is recorded, and a histogram of photon arrival times builds up as a number of optical pulses successively propagate through the setup. The camera is connected directly to a computer and we acquire data for the histograms during a 10-min period-this corresponds to 2 million identical pulses propagating through the setup. Use of a higher repetition rate laser $(\mathrm{MHz})$ would result in the acquisition time being reduced to less than $1 \mathrm{~s}$.

Figure 1 gives a schematic representation of the data cube $(x, y$ and $t$ ) acquired by the SPAD array. The histogram in Fig. 1 shows a 5-ns section for one of the pixels in the array: considering all of the $x, y$ locations at one particular time $t$ gives a snapshot of the spatial position of the laser pulse as shown for example in the individual time frames in Fig. 1. Integrating all time frames gives a static representation of the path followed by the light, similar to what is captured with an EMCCD camera, also shown in Fig. 1.

Data processing. The unique features of the three-dimensional data allow efficient data processing and noise suppression in both the temporal and spatial dimensions. The image processing algorithm is schematically illustrated in Fig. 2 that shows the timeintegrated image after each step of the data processing. Starting with the raw data, we first subtract the background data cube acquired in the same conditions, but without the laser propagating through the scene. Second, every time histogram is fitted with a Gaussian. For pixels containing signal from the laser pulse, this fitting removes what we could call the 'temporal noise', that is, the noise in the time bins with no signal from the laser pulse. Moreover, some spatial pixels are never illuminated by the laser pulse scatterings and contain only noise and background light and are thus set to zero. We therefore use the time information contained in each pixel to improve the spatial quality of our data. Third, we deconvolve the instrument impulse response function (largely due to electronic jitter and measured by uniformly illuminating the camera with a 100 -fs laser pulse) from the Gaussian fits. This results in histograms with a full-width at half-maximum of $\sim 500$ ps FWHM that is consistent with an independent measurement of the laser pulse duration. Finally, we increase the spatial quality of the images by a standard linear re-interpolation, frame by frame, between each line and column of the $32 \times 32$ pixel array as in Fig. 2. We interpolate in a way that connects the centre of mass of each line and column, in order to achieve a final spatial $310 \times 310$ pixel count. Summarizing, the data processing essentially relies on a three-stage process composed of noise removal, temporal deconvolution and re-interpolation: this process does not require any a priori knowledge of the scene content itself and is therefore inherently robust. The re-interpolation process is a very basic nearest-neighbour interpolation: no information is lost in this process and, naturally, no information is gained either. More importantly, all quantitative data analysis (for example, the plasma decay times as discussed below), is always performed on the raw (not interpolated) data.

Light propagation in air. The final result is shown in Fig. 3 where we show the evolution of the light pulse propagating through air. The figure shows selected time frames acquired by the camera, each separated by $0.7 \mathrm{~ns}$ and overlaid on a photograph (taken with a commercial DSLR camera) of the setup. The field of view of the SPAD camera is represented by dashed lines on each frame. In the first frame, the laser pulse has just entered the field of view. We then see the pulse propagate and reflecting

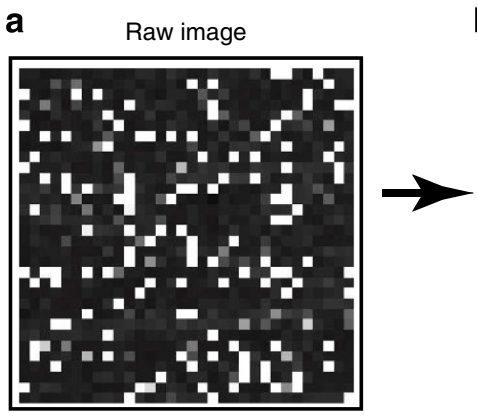

b Background subtracted image

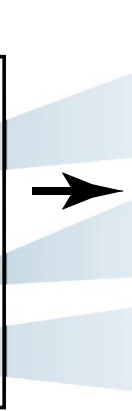

C Counts Time histograms
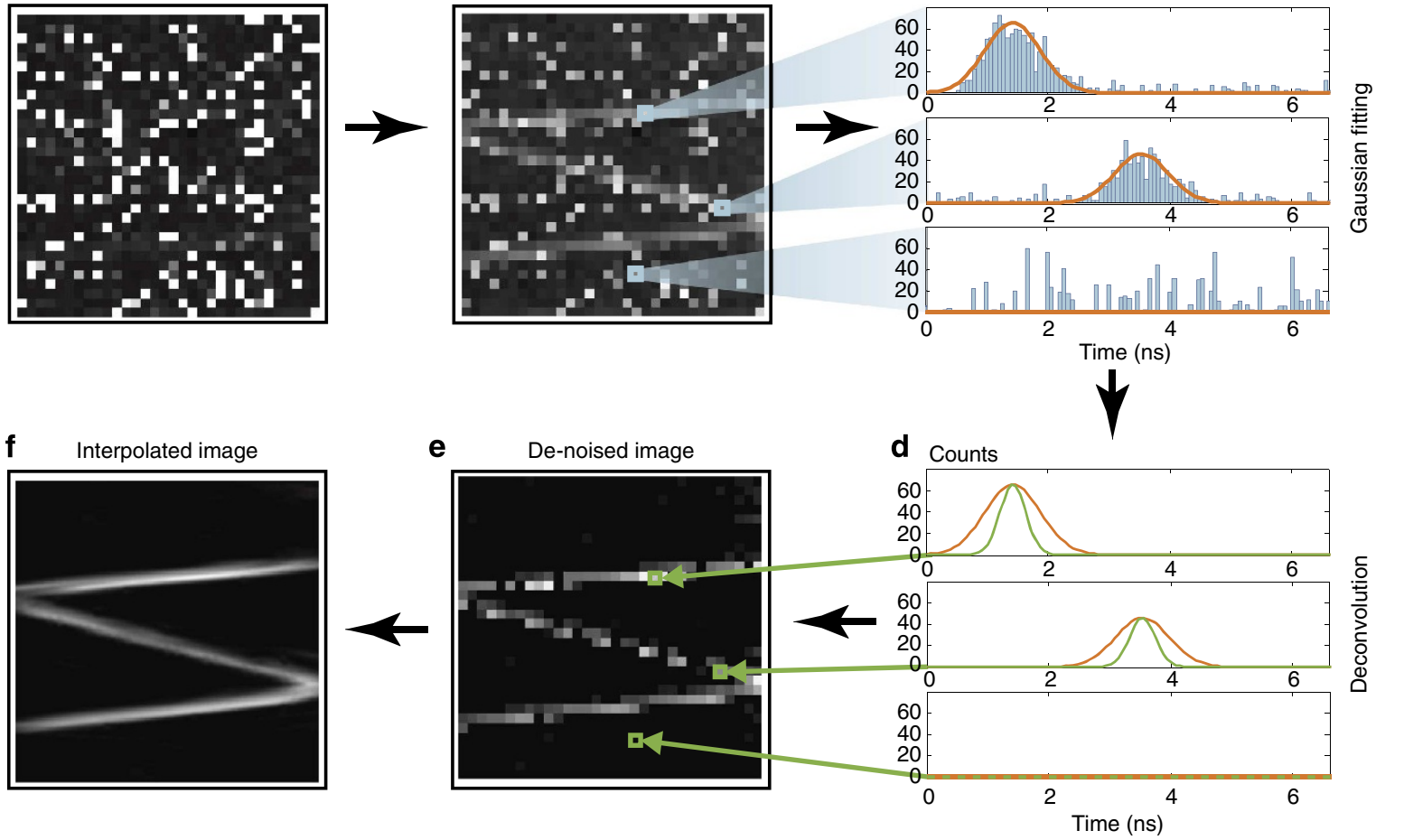

Figure 2 I Data processing. (a) To process the acquired data, we first subtract a background acquired by the SPAD camera without the laser beam propagating across its field of view. It allows us to see the path, but the image (b) is still very noisy. (c) To get a clearer image, we fit a Gaussian to every histogram. If the Gaussian is much wider than we expect, or smaller than the pulse duration, we set the histogram to zero. (d) We then deconvolve

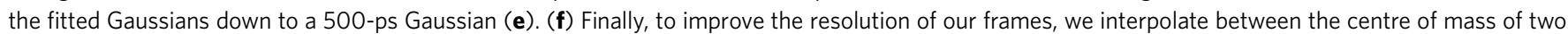
adjacent lines/columns. 


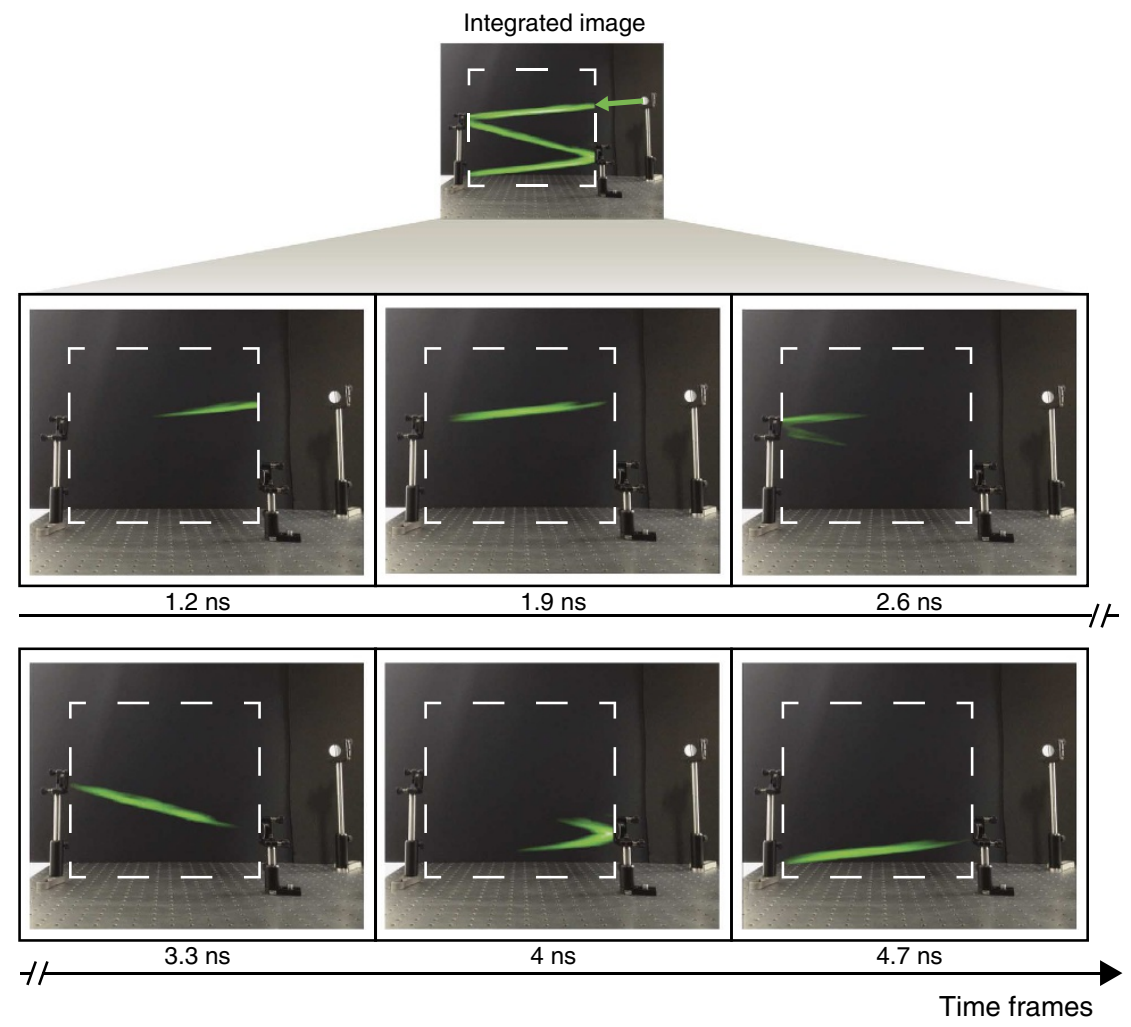

Figure 3 | Frames of the light-in-flight movie. In these frames, we see a pulse of light propagating between three mirrors. The laser first hits the mirror on the right and is directed towards the field of view of the SPAD camera, as indicated by the green arrow on the integrated image. The FOV is represented by dashed rectangles and corresponds to a $35 \times 35 \mathrm{~cm}^{2}$ region. In the first and second frames, we show the laser pulse entering the FOV. In the second, third and fourth frames, we see the light being reflected by the mirrors, before exiting the FOV in the last frame.

off the two mirrors in the subsequent frames, before finally leaving the field of view. A video of the full evolution of the laser pulse propagation is provided as Supplementary Video 1. The scene that we observed, that is, the pulse reflecting between the three mirrors, happens within a few nanoseconds, only a fraction of the $69 \mathrm{~ns}$ time range of the camera. During that time, the $500 \mathrm{ps}$ pulse, which is $15-\mathrm{cm}$ long, travels $67 \mathrm{ps}$ or $2 \mathrm{~cm}$ between each frame of the movie. The figure (and video) clearly demonstrates that we are able to capture the full dynamic of light propagation in air.

In these measurements, the average count rate from the detector is found to be of the order of 0.0005 detected events per laser pulse, per pixel. Even allowing for the relatively low fillfactor and single-photon detection efficiency, this implies a photon flux of the order of 0.2 photons per pulse, per full pixel area. The combination of single-photon sensitivity and picosecond temporal resolution make it very difficult for detector technologies such as intensified $\mathrm{CCD}^{19}$ to achieve the results shown.

Plasma dynamics. We also captured the dynamics of a more complex light phenomenon that is still the object of intense research studies for various applications: the formation of a weakly ionized plasma filament by a femtosecond laser pulse $\mathrm{e}^{20}$. Laser pulses with $90 \mathrm{fs}, 12 \mathrm{~mJ}$ (Amplitude Technologies), $785 \mathrm{~nm}$ wavelength at a $100-\mathrm{Hz}$ repetition rate were loosely focused with a $50-\mathrm{cm}$ lens to create a weak and tenuous plasma filament approximately $5 \mathrm{~cm}$ long, in the field of view of the SPAD camera. The weakly ionized plasma filament emits a faint fluorescence signal, largely in the $300-440 \mathrm{~nm}$ wavelength range ${ }^{21}$. The data were acquired for $2 \mathrm{~min}$ in two different spectral ranges using coloured filters: in red, to isolate the propagation of the laser pulse, and in blue, to isolate the plasma fluorescence. Figure 4 shows the scene for selected time frames (a full video is available as Supplementary Video 2). We first observe the femtosecond pulse (red) coming into the field of view and becoming brighter in the second frame as the pulse is scattered by the plasma created by its leading edge. The beam then moves out of the field of view, with a reduced intensity because of scattering from the selfinduced plasma. We can also see the plasma (blue) being formed and still fluorescing well after the beam has passed. The time histograms (an example is also shown in Fig. 4) reveal an exponential decay with a lifetime of approximately $600 \mathrm{ps}$, consistent with previous measurements ${ }^{20}$. It is important to note that the technique described here allows to characterize the plasma dynamics without the need to introduce any additional scattering agents, which would severely alter the plasma formation process itself.

\section{Discussion}

Our measurements open new possibilities for measuring ultrafast temporal dynamics within extremely low-intensity events. Remarkably, we are now able to observe light-in-flight at the sub-nanosecond scale in air where scattering occurs only from the ambient gas molecules. We underline that the SPAD camera not only allows to record a scene dynamically on very short-time scales, but it also provides an additional opportunity for noise reduction by exploiting the temporal dimension. Other applications are viable where the ability to record low photon numbers with high temporal resolution will enable viewing objects that are hidden from view, for example, looking around corners ${ }^{22-24}$ or through walls ${ }^{24-26}$. The single-photon sensitivity of our ultrafast 


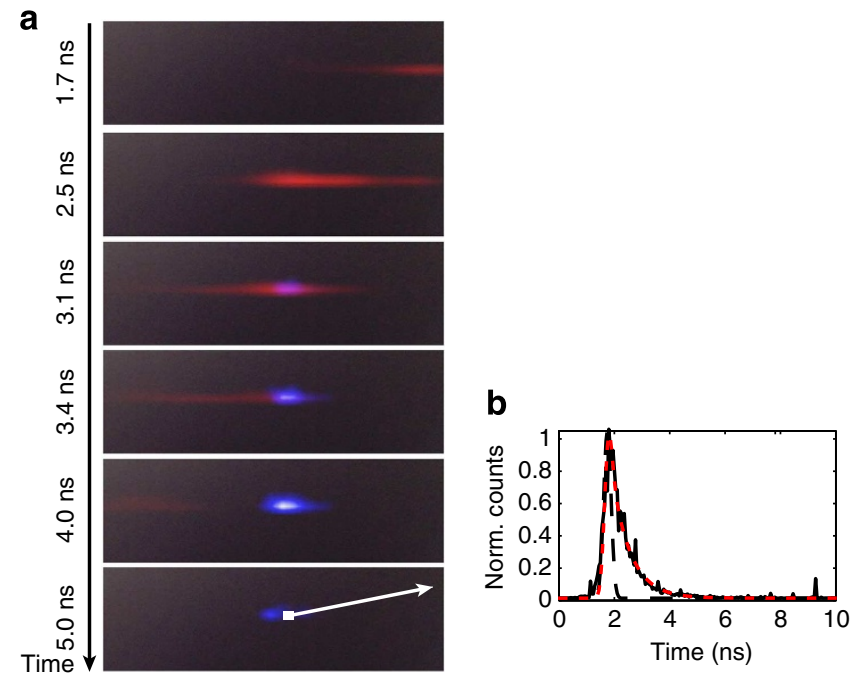

Figure 4 | Plasma evolution recorded by light-in-flight. (a) In these frames, the femtosecond pulse is shown in red, and the plasma fluorescence in blue. The plasma is created as soon as the leading edge of the pulse passes through focus. The laser pulse is then scattered by the plasma, which can be seen in the second frame. As a large portion of the beam is scattered or absorbed at the focus by the plasma, the exiting beam is weaker, as we observe in the third to fifth frames. We also see the plasma being formed at focus when the laser pulse is focused, then evolving and decaying after the pulse has passed. A full video is provided as Supplementary Video 2. (b) The graph shows the temporal histogram of the plasma fluorescence (solid black curve, normalized photon counts, raw data) corresponding to the region indicated with a white square in the last frame. The dashed line is the detector impulse response function, the dotted red curve is an exponential decay fit with a 600-ps decay constant.

imaging method enables direct spatially resolved measurements of dynamic quantum phenomena ${ }^{19}$, and also very weak scattering phenomena such as air-born acousto-optic effects ${ }^{27}$.

\section{Methods}

Details of experimental setup. The fisheye lens of $12-\mathrm{mm}$ focal length provides a $35-\mathrm{cm}$ wide field of view at the chosen object distance of $1.8 \mathrm{~m}$. The laser used to observe light reflecting off mirrors is a 532-nm wavelength microchip laser (Teem Photonics STG 03E), which emits 500 ps duration pulses of $3.5 \mu \mathrm{J}$ energy at $4 \mathrm{kHz}$ repetition rate. The laser polarization is set to vertical to maximize scattering in the direction of the camera. Experiments repeated in a pure nitrogen environment (data not shown) gave identical light intensities on the camera, thus verifying that the scattering is from air molecules and not from any possible dust particles that may be present in a standard atmospheric environment.

Time-correlated single-photon counting. The TCSPC timer of the SPAD array is operated in reverse mode, that is, it is started by the detection of a single photon, and stopped by the arrival of a periodic trigger. The trigger is derived from detection of a reflection of the laser pulse with an optical constant fraction discriminator (Becker \& Hickl OCF-401). The time range of the histogram acquired in TCSCP mode is $68.6 \mathrm{~ns}$ with a bin-width of $67 \mathrm{ps}$ (determined by the 10 bit acquisition electronics), and the median dark count rate of the pixels is 50 counts per second. In the experiment, the SPAD camera detects an average of 0.0005 photon per pulse per pixel, thus operating in the photon-starved regime as required for TCSPC.

\section{References}

1. Abramson, N. Light-in-flight recording by holography. Opt. Lett. 3, 121-123 (1978).

2. Abramson, N. Light-in-flight recording: high-speed holographic motion pictures of ultrafast phenomena. Appl. Opt. 22, 215-232 (1983).

3. Abramson, N. H. \& Spears, K. G. Single pulse light-in-flight recording by holography. Appl. Opt. 28, 1834-1841 (1989).

4. Häusler, G., Herrmann, J. M., Kummer, R. \& Lindner, M. W. Observation of light propagation in volume scatterers with 1011-fold slow motion. Opt. Lett. 21, 1087-1089 (1996)
5. Kubota, T., Komai, K., Yamagiwa, M. \& Awatsuji, Y. Moving picture recording and observation of three-dimensional image of femtosecond light pulse propagation. Opt. Express 15, 14348-14354 (2007).

6. Velten, A. et al. Femto-photography: capturing and visualizing the propagation of light. ACM Trans. Graph 32, 44:1-44:8 (2013).

7. Velten, A., Lawson, E., Bardagjy, A., Bawendi, M. \& Raskar, R. Slow art with a trillion frames per second camera. Proc. SIGGRAPH 44 (2011).

8. Heide, F., Hullin, M. B., Gregson, J. \& Heidrich, W. Low-budget transient imaging using photonic mixer devices. ACM Trans. Graph 32, 45:1-45:10 (2013).

9. Goda, K., Tsia, K. \& Jalali, B. Serial time-encoded amplified imaging for realtime observation of fast dynamic phenomena. Nature 458, 1145-1149 (2009).

10. Li, Z., Zgadzaj, R., Wang, X., Chang, Y.-Y. \& Downer, M. C. Single-shot tomographic movies of evolving light-velocity objects. Nat. Commun. 5, 3085 (2014).

11. O'Connor, D. \& Phillips, D. Time-Correlated Single Photon Counting (Academic, 1984).

12. Becker, W. Advanced Time-Correlated Single Photon Counting Techniques Vol. 81 (Springer, 2005).

13. Richardson, J. et al.A $32 \times 3250$ ps resolution 10 bit time to digital converter array in $130 \mathrm{~nm}$ cmos for time correlated imaging. Custom Integrated Circuits Conference, 2009. IEEE CICC '09, 77-80 (2009).

14. Li, D.-U. et al. Real-time fluorescence lifetime imaging system with a $32 \times 32$ cmos low dark-count single-photon avalanche diode array. Opt. Express 18, 10257-10269 (2010).

15. Buller, G. S. \& Wallace, A. Ranging and three-dimensional imaging using timecorrelated single-photon counting and point-by-point acquisition. IEEE J. Sel. Top. Quantum Electron. 13, 1006-1015 (2007).

16. Kirmani, A. et al. First-photon imaging. Science 343, 58-61 (2014).

17. Guerrieri, F., Tisa, S., Tosi, A. \& Zappa, F. Two-dimensional spad imaging camera for photon counting. IEEE Photon. J. 2, 759 (2010).

18. Richardson, J., Grant, L. \& Henderson, R. Low dark count single-photon avalanche diode structure compatible with standard nanometer scale cmos technology. IEEE Photon. Technol. Lett. 21, 1020-1022 (2009).

19. Fickler, R., Krenn, M., Lapkiewicz, R., Ramelow, S. \& Zeilinger, A. Real-time imaging of quantum entanglement. Sci. Rep. 3, 1914 (2013).

20. Couairon, A. \& Mysyrowicz, A. Femtosecond filamentation in transparent media. Phys. Rep. 441, 47-189 (2007).

21. Xu, H., Azarm, A., Bernhardt, J., Kamali, Y. \& Chin, S. The mechanism of nitrogen fluorescence inside a femtosecond laser filament in air. Chem. Phys. 360, 171 (2009).

22. Velten, A. et al. Recovering three-dimensional shape around a corner using ultrafast time-of-flight imaging. Nat. Commun. 3, 745 (2012).

23. Gupta, O., Willwacher, T., Velten, A., Veeraraghavan, A. \& Raskar, R. Reconstruction of hidden $3 \mathrm{~d}$ shapes using diffuse reflections. Opt. Express 20, 19096-19108 (2012).

24. Katz, O., Small, E. \& Silberberg, Y. Looking around corners and through thin turbid layers in real time with scattered incoherent light. Nat. Photon. 6, 459-553 (2012).

25. Freund, I. Looking through walls and around corners. Phys. A Stat. Mech. Appl. 168, 49-65 (1990).

26. Popoff, S., Lerosey, G., Fink, M., Boccara, A. C. \& Gigan, S. Image transmission through an opaque material. Nat. Commun. 1, 81 (2010).

27. Heshmat, B. et al. The effect of detector distance and beam width in acoustooptical beam deflection probing in air. J. Opt. L. Tech. 44, 734 (2012).

\section{Acknowledgements}

We acknowledge support from the European Research Council under the European Union's Seventh Framework Programme (FP/2007-2013)/ERC GA 306559, the Engineering and Physical Sciences Research Council (EPSRC, UK, Grants EP/J00443X/1, EP/ K03197X/1, EP/K015338), UK-STFC (grant ST/H005595/1), USAR Laboratory and USAR Office through the ISN (contract number W9911NF-13-D-0001) and ST Microelectronics, Imaging Division, Edinburgh, for their support in the manufacture of the Megaframe chip. The Megaframe project has been supported by the European Community within the Sixth Framework Programme IST FET Open.

\section{Author contributions}

G.G. performed the experiments and drafted the manuscript. R.H. designed the CMOS SPAD pixel architecture, N.K. provided support with CMOS SPAD array, C.L. and J.L. assisted with the experiments, R.R.T. and D.F. conceived the light-in-flight imaging work using the SPAD array camera. D.F. supervised and directed the project. All authors contributed to scientific discussions and to the final manuscript.

\section{Additional information}

Supplementary Information accompanies this paper at http://www.nature.com/ naturecommunications 
Competing financial interests: The authors declare no competing financial interests.

Reprints and permission information is available online at http://npg.nature.com/ reprintsandpermissions/

How to cite this article: Gariepy, G. et al. Single-photon sensitive light-in-flight imaging. Nat. Commun. 6:6021 doi: 10.1038/ncomms7021 (2015). (c) (i) This work is licensed under a Creative Commons Attribution 4.0 International License. The images or other third party material in this article are included in the article's Creative Commons license, unless indicated otherwise in the credit line; if the material is not included under the Creative Commons license, users will need to obtain permission from the license holder to reproduce the material. To view a copy of this license, visit http://creativecommons.org/licenses/by/4.0/ 


\section{DOl: $10.1038 /$ ncomms7408}

\section{Erratum: Single-photon sensitive light-in-flight imaging}

Genevieve Gariepy, Nikola Krstajić, Robert Henderson, Chunyong Li, Robert R. Thomson, Gerald S. Buller, Barmak Heshmat, Ramesh Raskar, Jonathan Leach \& Daniele Faccio

Nature Communications 6:6021 doi: 10.1038/ncomms7021 (2015); Published 27 Jan 2015; Updated 25 Feb 2015

The original version of this Article contained a typographical error in the spelling of 'flight' in the title of the paper. This has now been corrected in both the PDF and HTML version of the Article. 